\title{
Research on Servo System of Permanent Magnet Synchronous Motor Based on Model Design
}

\author{
Xin Jin $^{1, *}$, Tao Zhang ${ }^{2}$, Hualiang Zhang ${ }^{2}$, Peng Zeng ${ }^{2}$ and Anna Wang ${ }^{1}$ \\ ${ }^{1}$ College of Information Science and Engineering, Northeastern University, Shenyang, Liaoning Province, China \\ ${ }^{2}$ Key Laboratory of Industrial Control Network and System, Shenyang Institute of Automation, Chinese Academy of Sciences, \\ Shenyang, Liaoning Province, China \\ *Corresponding author
}

\begin{abstract}
The increasing demands of modern industry for motor control make the software more and more complex. The simulation platform and the hardware test platform in traditional control system of permanent magnet synchronous motor (PMS M) are often independent of each other. This paper aims to simplify the PMSM control system design and testing process. With the model-based design (MBD) method, it completes PMSM vector control from simple to complex and from the specific to the general. It builds control algorithm model by Simulink. With TMS320F28335 as the control chip, it completes the automatic generation of algorithm code through C2000 Support Package. In addition, it utilizes Code Composer Studio (CCS) to automatically compile and download the codes to the target control board, thus achieving the organic combination of simulation and hardware testing.
\end{abstract}

Keywords-permanent magnet synchronous motor, model based designed; hardware in loop

\section{INTRODUCTION}

As one of the main power equipment in modern society, the electric motor is widely applied in various fields, such as industry, agriculture, trans portation, national defense and so on. In literature 1, the motor is used to complete the lifting and traversing operations [1]. The simulation of control system is performed in Simulink. After the simulation has achieved the expected performance, the engineers need to prepare the $\mathrm{C}$ code manually, configure each register, etc. This development process has relatively high requirements of engineers [2, 3]. Besides, code development has low efficiency and huge difficulty.

The model-based design approach organically combines the four phases of mutual frag mentation in traditional development process. From the requirement analysis on the visual development platform (MATLAB), it begins to test and verify. This will allow engineers to focus their research on algorithms and test cases, leaving the programming of e mbedded real-time $\mathrm{C}$ code to be done automatically by the computer $[4,5]$. This will not only speed up the development of hardware and software, but also improve the reliability and consistency of the code.

This paper completes the permanent magnet synchronous motor vector control by using the model-based design method. It builds permanent magnet synchronous motor control system model through the Simulink, and completes the automatic conversion of the $\mathrm{C}$ model code to code with Embedded Coder toolbox. Finally, it generates and downloads $\mathrm{C}$ code. The code reliability is verified by TMDS320F28335 hardware and software tests. Software development can happen offline with hardware and software being developed in parallel, as depicted in Figure I.

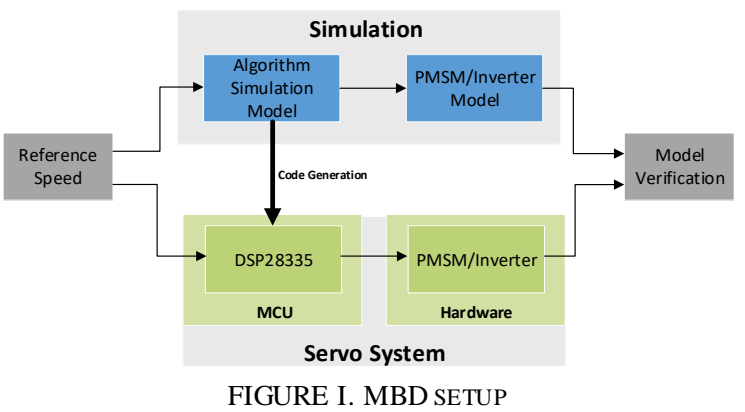

II SYSTEM MODELING AND SIMULATION

The experimental setup used in this paper is based on an AC feedback closed motor control system. The system represents a fully functional PMSM servo system with communication isolation, encoder feedback, and phase current sampling. At the core of the system is the Digital Signal Process or (DSP) from the TMDS320F28335. It completes the MBD platform deployment with tools from CCS and MathWorks.

\section{A. System Modeling Based on Simulink}

This paper adopts the vector control strategy of "id $=0$ ", and the control block diagram is shown in Figure II. The system uses dual closed-loop control of speed loop and current loop, when $\omega^{*}$ is used as the reference speed, and the output of the automatic speed regulator (ASR) is the q-axis current reference value $[6,7]$. And after the automatic current regulator (ACR), the output is $d, q$ axis voltage reference. Then it uses Space Vector Pulse Width Modulation (SVPWM) method to generate six PWM signals as the input of the inverter. 


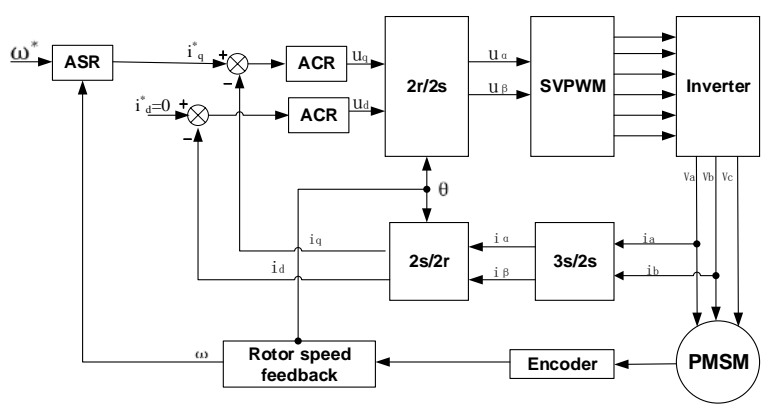

FIGURE II. PMSM VECTOR CONTROL BLOCK DIAGRAM

\section{B. Control Algorithm Modeling}

The algorithm model of the servo control system is established by building the Clark transformation module, the Park transformation module and the SVPWM module. The current regulator and the vector transformation part are showed in Figure III. $\theta$ represents the rotor flux linkage position. The stator currents called Ia and $\mathrm{Ib}$ are transformed into DC feedback value id and iq, then the current reference value compares with the current feedback value $[8,9]$. The current regulator output $\mathrm{d}$ axis voltage reference values and $\mathrm{q}$ axis voltage reference values called $U_{d}{ }^{*}$ and $U_{q}{ }^{*} . U_{d}{ }^{*}$ and $U_{q}{ }^{*}$ generate three-phase modulation wave $U_{a}{ }^{*}, U_{b}{ }^{*}, U_{c}{ }^{*}$ by inverse park transformation and SVPWM algorithm.

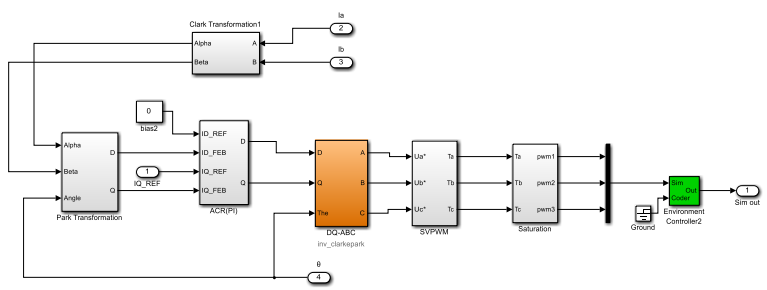

FIGURE III. CURRENT REGULATOR AND VECTOR CONVERSION

\section{Hardware Circuit Modeling}

SimPowerSystems toolbox module is used to complete the hardware circuit modeling and simulation. The experimental waveform effects could be observed through the scope module.

1) Three resistance current sampling and reconstruction simulation

The method of the current sampling in this article is a three-resistor current sampler. The three lower legs of the inverter are respectively connected in series with three resistance values of $0.02 \Omega$ sampling resistor to complete the three-phase current sampling. This method01 replaces the current sensor or Hall sensor, and achieves the purpose of reducing costs. The main circuit of the three-phase inverter composed of 6 Insulated Gate Bipolar Transistor (IGBT) modules. The three resistors named R1, R2 and R3 of the lower arm are the sampling resistors, as depicted in Figure IV.

In this case, the current sampled by the ADC module can accurately represent the three-phase current flowing through the motor only when the three lower arms, that is, IGBT2, IGBT4, and IGBT6 are turned on simultaneously. Therefore, current sampling is performed when IGBT2, IGBT4 and IGBT6 are turned on simultaneously. The voltage values of three devices are used to perform the AND operation to determine the time of simultaneous on, and the sampling current at the previous moment is maintained for the remaining time, so that the ADC module can always obtain the current value at the latest moment. Phase current sampling simulation model is shown in Figure IV.

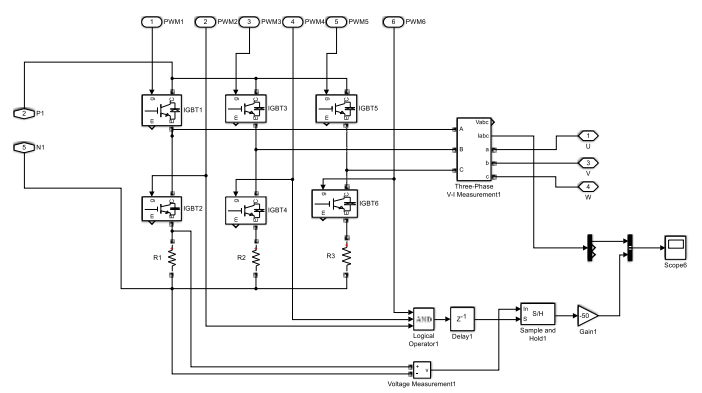

FIGURE IV. Three PhASE CURRENT SAMPLING AND RECONSTRUCTION

\section{System Simulation Model}

To obtain the simulation model of PMSM field-oriented vector control system, the above algorithm model is integrated with the hardware circuit model as depicted in Figure V. Scaling Module in the model includes the calculation of the speed loop and translates the standard value into the value of per unit. The collected signals include speed reference, speed feedback and current feedback. The current signal and the angle signal which were obtained through the feedback are input into the vector calculation model, then six pulse width modulation signals are output to drive the motor by the inverter module.

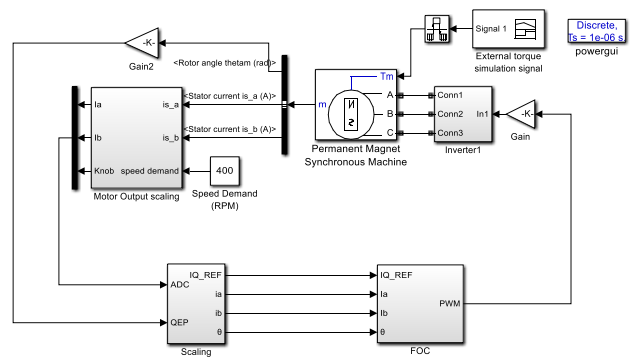

FIGURE V. Simulation MOdel of PMSM Vector CONTROL SySTEM

\section{SYSTEM MODELING AND CODE GENERATION}

\section{A. Algorithm Model and the Underlying Hardware-driven Integration}

This paper uses the TMDS320F28335 as a control chip, which is a high-performance digital signal processor specifically designed for motor control, and it can achieve floating-point arithmetic. The underlying hardware driver must be installed by adding Embedded Coder Support Package for the Texas Instruments C2000 Processors in the Simulink, which provides the QEP, ADC, PWM and other modules of the underlying hardware driver. The designer completes the setting of the peripheral parameters by double clicking on the underlying hardware driver module. This article sets the PWM frequency to $10 \mathrm{kHz}$ by filling the period value of the PWM module into the 7500 , and sets the dead time of 3 us by filling in the RED and FED value of 450. And this model acquires 
rotor angle by QEP module, which resulting in a count of 0 to 16000 to determine the rotor position. This model obtains the value of 'qposcnt' to determine the angle through QEP module. These settings will automatically generate peripheral module initialization code. The vector transformation model is shown in Figure VI.

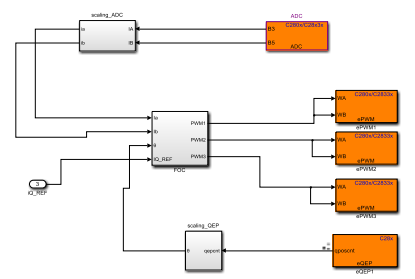

FIGURE VI. THE VECTOR TRANSFORMATION MODEL

\section{B. Three Resistance Current Sampling and Reconstruction}

It is necessary to sample the phase-current by using the sampling resistor when all the lower arms of the inverter are turned on. It is necessary to set the Event Trigger in the PWM module to "Enable ADC start of conversion for module A". The counting mode of the system is Up-Down, so the system start conversion for module when counter equals to period, as depicted in Figure VII. Three-phase insulated gate bipolar transistor (IGBT) intelligent power module is used as the inverter. Three-phase current sampling can be achieved when the three lower bridge arms are turned on simultaneously.

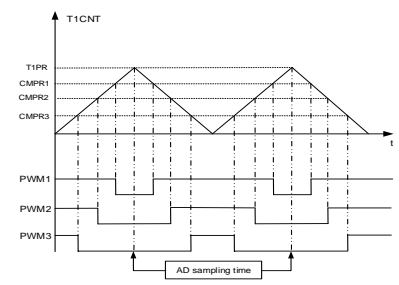

FIGURE VII. CURRENT SAMPLING TIMING DIAGRAM

\section{Simulation Model and the Underlying Hardware-driven Integration}

A standard field-oriented vector controller is implemented in Simulink, with auto-code generation being used to generate the controller modules in the Code Composer Studio-based C project. The simulation model is integrated with the underlying hardware-driven model.

The parameters of the motor in Simulink including voltage levels need to be as realistic as the actual motor. On the one hand, continuous simulations get the desired waveform, on the other hand, the simulation algorithm through the automatic generation of code to compile and download to the target control board get the desired waveform. The actual measured waveforms need to have almost the same effect as the simulation. Due to the existence between the simulation and the actual, the waveform cannot be exactly the same. The top of the graph is the simulation module of the hardware circuit. The modules such as PWM, QEP and ADC directly finish the configuration of the DSP peripheral so that the actual hardware can control the PMSM. This block is illustrated in Figure VIII.

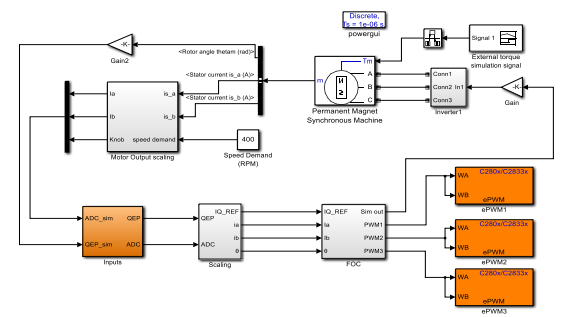

FIGURE VIII. VECT OR CONT ROL CODE AND SIMULATION MODEL

\section{SYSTEM OPERATION AND TESTING}

Prior to code generation, optimizations such as Model Advisor, Design Verifier, Model Validation, Software In Loop testing (SIL), Processor In Loop testing (PIL), code optimization, etc. can be performed to improve the efficiency of $\mathrm{C}$ code execution, and achieve satisfactory model coverage. After the optimization is completed, connect the DSP target board to the computer and click on the Build Model button on the Simulink interface to generate the servo control system code. In this process, MATLAB will prompt directly if there is any logic or statement error in the motor control system, without having to worry about the statement error of generating the code. If there is no problem, MATLAB will automatically generate the relevant code of PMSM control system, and the generated project files will be automatically opened in CCS software, the project files will be automatically compiled and automatically downloaded to the target board to complete the compilation and download process.

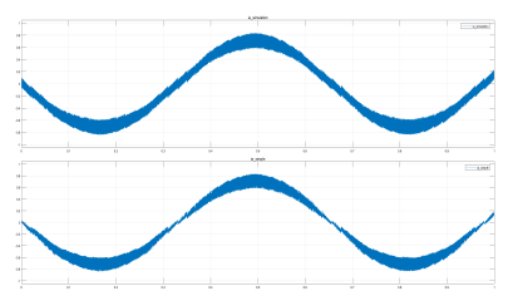

(a) SIMULATION OF PHASE CURRENT WA VEFORM

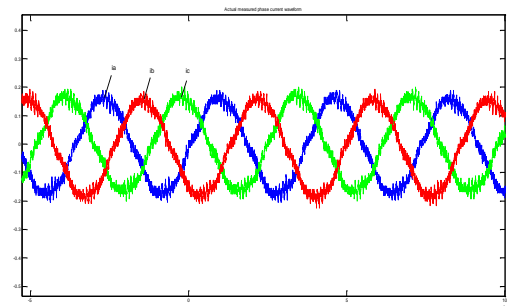

(b) ACTUAL MEASURED PHASE CURRENT WA VEFORM

FIGURE IX. SIMULATION AND ACTUAL MEASURED PHASE CURRENT WAVEFORM

In simulation, the actual current waveform is the same as the current waveform measured by the sampling resistor as depicted in Figure IX (a). The blue above represents the actual current and the lower line represents the sampled current. In the servo system, the phase currents flowing through the PMSM are sinusoidal signals differing by 120 degrees, as depicted in Figure IX (b). 


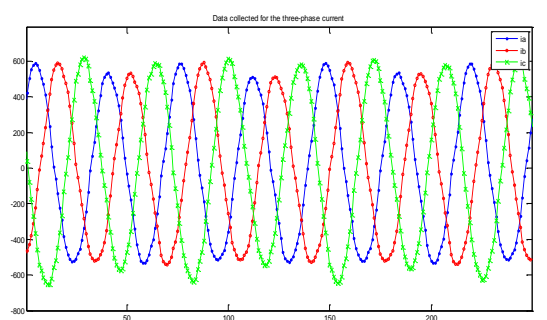

(a) DATA COLLECTED FOR THE THREE-PHASE CURRENT

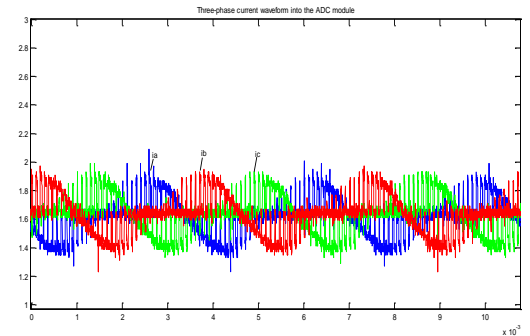

(b) THREE-PHASE CURRENT WAVEFORM INTO THE ADC MODULE

FIGURE X. PHASE CURRENT COLLECTED BY THE ADC AND PHASE CURRENT INPUT TO THE ADC

The conversion data of the ADC module is stored in the Memory Browser in the CCS software, and the stored data of the three-phase current is plotted using MATLAB as depicted in Figure $X(a)$. Figure $X(b)$ shows the actual three-phase current flowing into the ADC module. Through quantitative calculation, the collected current data and the actual current data are consistent.

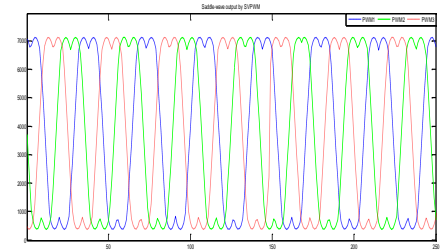

(a) Saddle-wave output by SVPWM

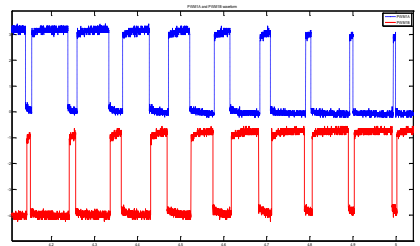

(b) PWM1A and PWM1B waveform

FIGURE XI. INPUT WA VEFORM AND OUTPUT WA VEFORM OF PWM MODULE

The SVPWM module generates saddle-waves to control PWM modules. The saddle-wave data is also recorded using the CCS software and plotted using MATLAB, as depicted in Figure XI (a). The duty cycle of PWM changes linearly. The blue line represents the waveform of PWM1A, the other represents the PWM1B as shown in Figure XI (b).

It can be seen from the experimental waveforms that the actual waveform of the system is in good accordance with the simulation waveforms. The experimental results are in line with the theoretical analysis and engineering requirements, which fully demonstrates the feasibility and efficiency of model-based design in developing the motor control codes.

\section{CONCLUSION}

In this paper, vector control of PMSM is implemented by using the model-based design method. Phase current sampling and reconstruction are realized by three sampling resistors. The current sensor is effectively replaced and a simulation model of three-resistor current sampling is established. The sampling time of the ADC module is set to the moment when the lower arms of the inverter turn on at the same time. It is verified that the simulation effect is obvious, and the current waveform sampled by the ADC module is consistent with the actual current waveform.

Meanwhile, the simulation model of servo control system and the underlying hardware-driven code model are established. The code model is automatically converted into effective $\mathrm{C}$ code by combining MATLAB and CCS, and tested on the hardware platform to quickly complete the system modeling and achieve the vector control of PMSM. The results of the test verify the correctness of the model and the validity of the code, but also reduce the difficulty of development, significantly improve the development efficiency.

\section{ACKNOWLEDGEMENTS}

This work was partially supported by National Natural Science Foundation of China under Grant (NO.91648204); National Science and Technology Major Project under Grant (NO.2017ZX02101007-004).

\section{REFERENCES}

[1] X. Jin. S. Wang. Stereo Garage Demo Platform by Lifting and Parallel Moving Based on Embedded Technology. J. Value Engineering.2015, 34(03):214-215.

[2] G. Nicolescu. P. J. Mosterman. Model-Based Design for Embedded Systems. U.S. 2010.

[3] SAOUD I M, BEN S. Model-based design approach for embedded digital controllers design. J. International Journal of Automation and Control, 2011, 5(3):267-283.

[4] O'SULIVAN D, SORENSEN J, FREDERIKSEN A. Model based design tools in loop motor control. C. International Exhibition and Conference for Power, 2014:1643-1651.

[5] WANG L M, WANG G N, WANG W, et al. MBD-DSP: A Model Based Design Solution for DSP. C. International Conference on Electrical and Control Engineering. IEEE, 2011; 4561-4564.

[6] T. Zhang. L. Cui, H. Li, F. Wang and A. Wang, "A method of motor's absolute position detection based on the single fiber bi-directional technology," 2014 IEEE International Conference on Information and Automation (ICIA), Hailar, 2014, pp. 644-647.

[7] F. Y. Wang. Research on the Control System of Permanent Magnet Synchronous Motor Based on High-frequency Signal Injection. D. Northeastern University, 2014.

[8] T. Zhang. The Sensorless Control of Permanent Magnetic Synchronous Motor (PMSM). D. Dalian University of Technology.2011.

[9] T. Zhang, Z.Q. Yang. X. D. Wang. Method of Measuring Motor Position and Speed with eQEP Unit and Encoder. J. Electric Drive, 2011, 41(04):48-51. 